\title{
DEFORMABILITY OF A SUBMANIFOLD IN A EUCLIDEAN SPACE WHOSE IMAGE BY THE GAUSS MAP IS FIXED
}

\author{
YOSIO MUT̄̄
}

\begin{abstract}
In the present paper, deformations of an immersion of an $m$-dimensional manifold $M$ in Euclidean $n$-space $R^{n}$ such that the Gauss image is fixed pointwise are studied. We call such deformations admissible deformations. The main theorem gives a necessary and sufficient condition for an immersed manifold to admit nontrivial admissible deformations.
\end{abstract}

1. Let $M$ be an $m$-dimensional $C^{\infty}$ manifold and $i: M \times I \rightarrow R^{n}$ a $C^{\infty}$ immersion where $I$ is some interval, $0 \in I$. $i(t)$ is defined by $i(t) M=i(M \times$ $t)$ and we write $i(t) M=M(t) . M(t)$ is called a deformation of $M(0)$. We assume the Gauss map $\Gamma: M(t) \rightarrow G(m, n-m)$ is regular for $t \in I$. Let $M(t)$ have the local expression

$$
x^{h}=x^{h}\left(y^{1}, \ldots, y^{m} ; t\right)
$$

where $x^{1}, \ldots, x^{n}$ are the rectangular coordinates in $R^{n}$ and $y^{1}, \ldots, y^{m}$ are local coordinates in $M$. We understand

$$
\begin{aligned}
& \partial_{i} \Phi=\frac{\partial \Phi}{\partial x^{i}}, \quad \partial_{\lambda} \Phi=\frac{\partial \Phi}{\partial y^{\lambda}}, \quad \dot{\Phi}=\Phi=\frac{\partial \Phi}{\partial t}, \\
& h, i, j, \ldots=1, \ldots, n ; \quad \kappa, \lambda, \mu, \ldots=1, \ldots, m .
\end{aligned}
$$

Suppose there exists a $(1,1)$-tensor field $a(y ; t)$ satisfying

$$
\partial_{\lambda} \dot{x}^{h}=a_{\lambda}{ }^{o} \partial_{\sigma} x^{h}
$$

Obviously the geometrical meaning of this equation is that the Gauss image of $M(t)$ is fixed against $t$ for each point of $M$. Let us call any such deformation $M(t)$ an admissible deformation in this paper. As usual let $g_{\mu \lambda}$ be the compo-

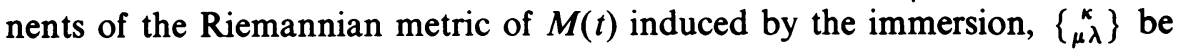
the Christoffel symbols, $\nabla$ be covariant differentiation with respect to the Christoffel symbols, $B_{\mu}{ }^{h}=\partial x^{h} / \partial y^{\mu}$ and $H_{\mu \lambda}{ }^{h}$ be the second fundamental tensor.

First we get from (1.2):

$$
\partial_{\mu} \partial_{\lambda} \dot{x}^{h}-\left\{\begin{array}{c}
\sigma \\
\mu \lambda
\end{array}\right\} \partial_{\sigma} \dot{x}^{h}=\nabla_{\mu} a_{\lambda}{ }^{o} B_{\sigma}{ }^{h}+a_{\lambda}{ }^{\sigma} H_{\mu \sigma}{ }^{h}
$$

Received by the editors September 8, 1978.

AMS (MOS) subject classifications (1970). Primary 53B25, 53C40.

Key words and phrases. Submanifold of Euclidean space, deformation with fixed Gauss image, admissible deformation. 
Then in view of the identity $\Sigma_{h} B_{\nu}{ }^{h} H_{\mu \lambda}{ }^{h}=0$ we get

$$
\begin{gathered}
\nabla_{\mu} a_{\lambda}{ }^{\kappa}=\nabla_{\lambda} a_{\mu}{ }^{\kappa}, \\
a_{\mu}{ }^{\sigma} H_{\lambda \sigma}{ }^{h}=a_{\lambda}{ }^{\sigma} H_{\mu \sigma}{ }^{h} .
\end{gathered}
$$

Differentiating $g_{\mu \lambda}=\Sigma_{h} \partial_{\mu} x^{h} \partial_{\lambda} x^{h}$ with respect to $t$ we get

$$
\dot{g}_{\mu \lambda}=a_{\mu \lambda}+a_{\lambda \mu}
$$

where $a_{\mu \lambda}=a_{\mu}{ }^{\sigma} g_{\sigma \lambda}$. From this and

$$
\frac{\partial}{\partial t}\left(\partial_{\nu} g_{\mu \lambda}-\left\{\begin{array}{c}
\kappa \\
\nu \mu
\end{array}\right\} g_{\kappa \lambda}-\left\{\begin{array}{c}
\kappa \\
\nu \lambda
\end{array}\right\} g_{\kappa \mu}\right)=0
$$

we get

$$
\left(\nabla_{\nu} a_{\mu}{ }^{\kappa}-\left\{\begin{array}{c}
\kappa \\
\nu \mu
\end{array}\right\}^{\cdot}\right) g_{\kappa \lambda}+\left(\nabla_{\nu} a_{\lambda}{ }^{\kappa}-\left\{\begin{array}{c}
\kappa \\
\nu \lambda
\end{array}\right\}^{\cdot}\right) g_{\kappa \mu}=0 .
$$

This shows that the tensor defined by

$$
A_{\nu \mu \lambda}=\left(\nabla_{\nu} a_{\mu}{ }^{\kappa}-\left\{\begin{array}{c}
\kappa \\
\nu \mu
\end{array}\right\}\right) g_{\kappa \lambda}
$$

is skew symmetric in $\mu$ and $\lambda$. On the other hand, $A_{\nu \mu \lambda}$ is symmetric in $\nu$ and $\mu$ in view of (1.3). Hence we have

$$
\left\{\begin{array}{c}
\kappa \\
\mu \lambda
\end{array}\right\}^{\cdot}=\nabla_{\mu} a_{\lambda}{ }^{\kappa}
$$

Differentiating

$$
H_{\mu \lambda}{ }^{h}=\partial_{\mu} \partial_{\lambda} x^{h}-\left\{\begin{array}{c}
\kappa \\
\mu \lambda
\end{array}\right\} \partial_{\kappa} x^{h}
$$

and the Gauss equation

$$
K_{\nu \mu \lambda \kappa}=\sum_{h} H_{\nu \kappa}{ }^{h} H_{\mu \lambda}{ }^{h}-\sum_{h} H_{\mu \kappa}{ }^{h} H_{\nu \lambda}{ }^{h}
$$

with respect to $t$ and taking (1.6) into account, we get

$$
\begin{gathered}
\dot{H}_{\mu \lambda}{ }^{h}=a_{\lambda}{ }^{\sigma} H_{\mu \sigma}{ }^{h}, \\
\dot{K}_{\nu \mu \lambda \kappa}=a_{\lambda}{ }^{\sigma} K_{\nu \mu \sigma \kappa}+a_{\kappa}{ }^{\sigma} K_{\nu \mu \lambda \sigma} .
\end{gathered}
$$

2. From (1.8) we get:

TheOREM 2.1. Assume in an admissible deformation $M(t)$ that $M(0)$ is a flat Riemannian manifold. Then $M(t)$ is a flat Riemannian manifold.

From (1.5) and (1.6) we can easily prove:

TheOREM 2.2. Assume in an admissible deformation $M(t)$ that $t$ induces an isometric deformation of $M(t)$. Then the tensor field $a_{\mu}^{\lambda}$ is covariantly constant and $a_{\mu \lambda}=-a_{\lambda \mu}$.

If an admissible deformation $M(t)$ is such that the tensor field $a$ vanishes, then $M(t)$ undergoes only parallel displacements. If $\left(a_{\mu}{ }^{\lambda}\right)$ is a scalar matrix, then $M(t)$ changes homothetically. In such cases the admissible deformation is said to be trivial. 
Let $i_{0}: M \rightarrow R^{n}$ be a $C^{\infty}$ immersion. If there exists a nontrivial admissible deformation $M(t)$ such that $M(0)=i_{0} M$, then $M(0)$ is said to be nontrivially deformable with the Gauss image fixed, or to admit a nontrivial admissible deformation.

A typical nontrivial deformation with fixed Gauss image is found in the case of $n=m+1$, as is well known.

REMARK 1. If $n=m+1$, we have for any deformation $\dot{x}^{h}=\xi N^{h}+t^{\lambda} B_{\lambda}{ }^{h}$ where $N$ is the unit normal vector field, $\xi$ is a function on $M$ and $t^{\lambda}$ is a vector field on $M$. Then we get

$$
\partial_{\mu} \dot{x}^{h}=\left(\nabla_{\mu} \xi+t^{\lambda} h_{\mu \lambda}\right) N^{h}+\left(-\xi h_{\mu}{ }^{\lambda}+\nabla_{\mu} t^{\lambda}\right) B_{\lambda}{ }^{h} .
$$

(1.2) is satisfied if we take $t^{\lambda}$ such that $\nabla_{\mu} \xi+t^{\lambda} h_{\mu \lambda}=0$ and put $a_{\mu}{ }^{\lambda}=\nabla_{\mu} t^{\lambda}-$ $\xi h_{\mu}{ }^{\lambda}$.

A product immersion also admits a nontrivial admissible deformation.

REMARK 2. By a product immersion we mean the case $M=M_{1} \times$ $M_{2}, i(t) M=i_{1}(t) M_{1} \times i_{2}(t) M_{2}$. Then a nontrivial admissible deformation is obtained if we assume $i_{1}(t) M_{1}$ and $i_{2}(t) M_{2}$ are homothetic to $i_{1}(0) M_{1}$ and $i_{2}(0) M_{2}$, respectively.

3. We prove the following main theorem.

THeOREM 3.1. Let $M$ be a compact $C^{\infty}$ manifold and let $M_{0}=i_{0} M$ be a $C^{\infty}$ immersion into $R^{n}$ expressed locally by $x^{h}=\varphi^{h}\left(y^{1}, \ldots, y^{m}\right)$ where the $x^{h}$ $(h=1, \ldots, n)$ are rectangular coordinates and $y^{\kappa}(\kappa=1, \ldots, m)$ are local coordinates of $M$. Let $b_{\lambda}{ }^{k}$ be a $(1,1)$-tensor field on $M$ such that there exists no constant $c$ satisfying $b_{\lambda}{ }^{\alpha}=c \delta_{\lambda}{ }^{\kappa}$ and consider the following conditions:

(i) $b_{\lambda}{ }^{k}$ satisfies the equations

$$
\nabla_{\mu} b_{\lambda}{ }^{\kappa}=\nabla_{\lambda} b_{\mu}{ }^{\kappa}, \quad b_{\mu}{ }^{\sigma} H_{\lambda \sigma}{ }^{h}=b_{\lambda}{ }^{\sigma} H_{\mu \sigma}{ }^{h}
$$

where the Riemannian connection is induced by the immersion $i_{0}$;

(ii) there exist functions $V^{h}(h=1, \ldots, n)$ satisfying

$$
\partial_{\mu} V^{h}=b_{\mu}{ }^{\sigma} \partial_{\sigma} \varphi^{h}
$$

Then (a) condition (i) follows from (ii); (b) if $M$ is simply connected, (i) is equivalent to (ii); (c) a necessary and sufficient condition that the immersion $M_{0}$ admits a nontrivial admissible deformation is that there exists a tensor field $b_{\lambda}{ }^{k}$ satisfying the condition (ii); (d) especially when $M$ is simply connected, $M_{0}$ admits a nontrivial admissible deformation if there exists a tensor field $b_{\lambda}{ }^{k}$ satisfying (i).

Proof. (a) is obtained if we substitute (3.2) into $\partial_{\mu} \partial_{\lambda} V^{h}-\partial_{\lambda} \partial_{\mu} V^{h}=0$. As the $b_{\mu}{ }^{\sigma} \partial_{\sigma} \varphi^{h}$ are closed forms on $M$ if (3.1) holds, (b) follows immediately. (d) is obtained from (b) and (c). Hence we need only to prove (c) and especially the sufficiency, for (3.2) is a special case of (1.2).

For that purpose first consider the system of ordinary differential equations 


$$
d a_{\lambda}{ }^{\kappa} / d t+a_{\lambda}{ }^{\rho} a_{\rho}{ }^{\kappa}=0
$$

with the initial condition $a_{\lambda}{ }^{\kappa}(0)=b_{\lambda}{ }^{\kappa}$ at each point $p$ of $M$. Let $A=\left(a_{\lambda}{ }^{\kappa}\right)$ and $B=\left(b_{\lambda}{ }^{k}\right)$. Then the solution is

$$
A=(E+B t)^{-1} B
$$

where $E$ is the unit matrix and the domain of $t$ is a certain interval $\left(-\varepsilon_{p}, \varepsilon_{p}\right)$. $M$ being compact, there exists a positive number $\varepsilon$ such that $\varepsilon<\varepsilon_{p}$ for all $p \in M$. If we consider at each point of $M$ the system of ordinary differential equations

$$
d \beta_{\mu}{ }^{h} / d t=a_{\mu}{ }^{\sigma}(t) \beta_{\sigma}{ }^{h},
$$

there exists also a unique solution $\beta_{\mu}{ }^{h}(t)$ satisfying the initial condition

$$
\beta_{\mu}{ }^{h}(0)=\partial_{\mu} \varphi^{h}
$$

From (3.3) and (3.4) we get $d^{2} \beta_{\mu}{ }^{h} / d t^{2}=0$ and the solution of (3.4) is

$$
\beta_{\mu}{ }^{h}=\beta_{\mu}{ }^{h}(0)+a_{\mu}{ }^{\sigma}(0) \beta_{\sigma}{ }^{h}(0) t \text {. }
$$

Then in view of (3.2) and (3.5) we get

$$
\beta_{\mu}{ }^{h}=\partial_{\mu}\left(\varphi^{h}(y)+V^{h}(y) t\right)
$$

and the immersion given by

$$
x^{h}(y ; t)=\varphi^{h}(y)+V^{h}(y) t
$$

satisfies

$$
\partial^{2} x^{h}(y ; t) / \partial y^{\lambda} \partial t=\partial_{\lambda} V^{h}
$$

On the other hand we get, from (3.6) and (3.7), $a_{\mu}{ }^{\sigma} \partial_{\sigma} x^{h}(y ; t)=a_{\mu}{ }^{\sigma} \beta_{\sigma}{ }^{h}$ which becomes on account of (3.4) and (3.6):

$$
a_{\mu}{ }^{\sigma} \partial_{\sigma} x^{h}(y ; t)=\partial \beta_{\mu}{ }^{h} / \partial t=\partial_{\mu} V^{h}
$$

From this result and (3.8) we get $\partial^{2} x^{h} / \partial y^{\lambda} \partial t=a_{\lambda}{ }^{\sigma} \partial_{\sigma} x^{h}$ and (c) is proved. Q.E.D.

We want to give an application. The space of $(1,1)$-tensor fields $b_{\lambda}{ }^{\kappa}$ satisfying (3.1) is a vector space over $R$ if the case $b_{\lambda}{ }^{\kappa}=c \delta_{\lambda}^{\kappa}$ is not excluded. Let us denote it by $\mathfrak{b}$. Let $l=\operatorname{dim} \mathfrak{b}$ and $h=\operatorname{dim} H^{1}(M)$. Then there exists a subspace $\mathfrak{b}_{1}$ of $\mathfrak{b}$ of dimension at least $l-h$ such that $b_{\lambda}{ }^{\sigma} \partial_{\sigma} x^{1}$ is a gradient for each tensor field $b_{\lambda}{ }^{\kappa}$ of $\mathfrak{b}_{1}$. There exists also a subspace $\mathfrak{b}_{2}$ of $\mathfrak{b}_{1}$ of dimension at least $l-2 h$ such that $b_{\lambda}{ }^{\sigma} \partial_{\sigma} x^{2}$ is a gradient for each element $b_{\lambda}{ }^{\kappa}$ of $\mathfrak{b}_{2}$ and so on. Thus, if $l-n h-1>0$ holds, there exists a tensor field $b_{\lambda}{ }^{k}$ satisfying (ii) of Theorem 3.1. This proves:

TheOrem 3.2. If $l=\operatorname{dim} \mathfrak{b}$ and $h=\operatorname{dim} H^{1}(M)$ satisfy $l-n h-1>0$, then the immersion $i_{0} M$ admits a nontrivial admissible deformation.

4. Let $M$ be a Veronese manifold immersed in a Euclidean space $R^{n}$ in full. If $H_{\mu \lambda}{ }^{h}$ is the second fundamental tensor at a point $p$ of $M$, then for any vector $u^{h}$ of $R^{n}$ satisfying 


$$
\sum_{h} H_{\mu \lambda}{ }^{h} u^{h}=0
$$

we have [1]

$$
u^{h}=t^{\lambda} B_{\lambda}{ }^{h} \text {. }
$$

As we have $n=m(m+3) / 2$ for the Veronese manifold, the above mentioned fact indicates that there exist among $n$ symmetric $(m \times m)$ matrices $\left(H_{\mu \lambda}{ }^{h}\right)$ just $m(m+1) / 2$ linearly independent symmetric matrices. This proves that the matrix $\left(a_{\mu}{ }^{\lambda}\right)$ satisfying (1.4) is a scalar matrix. Thus we have

THEOREM 4.1. A Veronese manifold immersed in a Euclidean space in full admits no nontrivial admissible deformation.

This means that, when a Veronese manifold immersed in a Euclidean space in full undergoes a deformation other than a parallel displacement, a homothety, or their composition, then the Gauss image moves pointwise.

5. Let us consider an immersion $i_{0}: M \rightarrow R^{n}$ and an admissible deformation $M(t), M(0)=i_{0} M$, again. Let $H_{\mu \lambda}{ }^{h}$ be the second fundamental tensor of $M(t)$ at $i(t) p$ where $p$ is fixed. When $\mu$ and $\lambda$ are given and $h$ runs the range $1, \ldots, n$ we get components of a vector of $R^{n}$. There are $m^{2}$ such vectors and they span a vector space whose dimension we denote by $d_{p}$. Then $d_{p}$ satisfies $d_{p}<m(m+1) / 2$ and $d_{p} \leqslant n-m$. In view of (1.7) we get:

THEOREM 5.1. $d_{p}$ is an invariant of any admissible deformation.

\section{BIBLIOGRAPHY}

1. Y. Muto, Submanifolds of a Euclidean space with homothetic Gauss map, J. Math. Soc. Japan (to appear).

2262-150, TOMOKA-CHO, Kanazawa-KU, YOKOHAMA 236, JAPAN 